\title{
Ultra-high temperature Raman-based distributed optical fiber sensor with gold-coated fiber
}

\author{
Ismail Laarossi, Ruben Ruiz-Lombera, Maria Angeles Quintela, Jesus Mirapeix, Domingo Lima, David Solana \\ and Jose-Miguel Lopez-Higuera Senior Member, IEEE
}

\begin{abstract}
An ultra-high temperature distributed sensor based on a Raman Optical-Time-Domain-Reflectometry (ROTDR) and two types of fibers: a standard multimode fiber and multimode gold-coated fiber are experimentally validated in this paper. A calibration technique has been implemented to correct the dynamic variation of the optical loss in the gold-coated fiber. Distributed temperature measurements up to $600^{\circ} \mathrm{C}$ have been carried out.
\end{abstract}

Index Terms - ultra-high temperature, distributed sensor, multimode gold-coated fiber, Optical-Time-Domain-Reflectometry, Raman DTS.

\section{INTRODUCTION}

O PTICAL fiber distributed sensors are able to provide measurements of the magnitude value along the measurement space. The result is given as a continuous function of position along the optical fiber sensor. This allows the monitoring in multiple points and can be performed with a single fiber. As a result, it avoids the strategic placement of multiple point conventional discrete sensors [1]. This inherent advantage, combined with the known characteristics of optical fibers such as the immunity to electromagnetic interference, and their ability to work in hostile environments [2], [3] have made the optical fiber distributed sensors systems an intense research area in the last few years.

Using the optical effects that occur along the optical fibers, it is possible to develop systems for measuring multiple physical parameters, such as strain, temperature, electromagnetic field and so on [4]. Most of the optical fiber distributed sensor systems use the scattering process to determine some of these parameters. These sensors are based on different effects such as Rayleigh scattering, spontaneous or stimulated Brillouin scattering and spontaneous Raman scattering [5].

Temperature is one of the most controlled physical parameters in industrial processes. Due to its low complexity and insensitivity to strain, distributed temperature sensor (DTS) systems based on spontaneous Raman scattering in optical fiber are expanding rapidly in the industry. Nowadays, this

I.laarossi, R.Ruiz-Lombera, M.A.Quinetla, J.Mirapeix and J.M.LopezHiguera are with the Photonics Engineering Group of Universidad de Cantabria, Santander, 39005, Spain. e-mail: ismail.laarossi@unican.es

D. Lima and D. Solana are with ENSA, Equipos Nucleares S.A, Avda. Juan Carlos I, 8, 39600 Maliaño (Cantabria-Spain). technology is in a mature state for some industrial applications such as thermal control of power lines, especially those of high and medium voltage [6], fire detection in tunnels [7] and buildings and leak detection in oil wells [8].

Most Raman-based DTS systems and fiber sensors are designed for operation up to approximately $300^{\circ} \mathrm{C}$. However, high-temperature distributed sensing for temperatures up to about $600^{\circ} \mathrm{C}$ is a very interesting application in the industry field. For example, to improve the energy output in a geothermal well, recent researches have the purpose to produce geothermal fluids at temperatures up to $550-600^{\circ} \mathrm{C}$ [9].

The limiting factor in these applications, when a high temperature monitoring is required, is the optical fiber for the distributed fiber transducer. It should be able to maintain its physical and optical properties after being exposed to these temperatures. Optical fibers are generally protected with a resistant polymer coating (such as polyamide), which provides sufficient protection at low temperatures. However, for temperatures higher than $300^{\circ} \mathrm{C}$, this type of coating can not be used, because the cover is completely burned and disappears, becoming the fiber fragile and easy to break. This prevents its use in real environments. Therefore, it is necessary to employ fibers with other coatings that can withstand these high temperatures such as metal coatings (aluminum or gold depending on the application and the maximum temperature to be reached) [10]. Although these fibers are expensive compared to standard fibers, they can be used again after being subjected to high temperatures, exhibiting a higher mechanical strength. This makes these fibers the best option for different industrial scenarios.

The problem that arises with the use of the metal-coated fibers in Raman distributed sensors systems is the dynamic changes and the non-uniform loss profile [11]. This fact implies that conventional calibration techniques can not be employed. The use of a single calibration after installation and connection of the optical fiber does not give correct results in these cases. A calibration technique that effectively compensates the non-linear differential attenuation characteristics of the fiber is required.

Previous works have reported temperature measurements from $25^{\circ} \mathrm{C}$ to $600^{\circ} \mathrm{C}$ [12]. Mandal et al., (2015) used a complex system to achieve high and stable temperatures. Moreover, the work is focused on the modification of the electronic part of the system (changing the gain of the photodetector as a function of the received signal). In our submission, an easy system with the ability to auto-configure itself is proposed, regardless of the fiber configuration and the 
temperature to measure.

In this paper we present a high temperature distributed sensor system (up to $600^{\circ} \mathrm{C}$ ) using a Raman Optical-TimeDomain-Reflectometry (ROTDR) system and two types of fibers: a standard multimode fiber and multimode gold-coated fiber. In addition, a comparison between these fibers have been realized. Several measurements at different temperatures have been made in order to show the problems that arise when temperature measurements are carried out with the gold-coated fiber. Finally, a calibration technique has been implemented to correct the errors in the measurements caused by the dynamic variation of the optical loss with temperature in the Stokes and the anti-Stokes wavelengths, which allows deployment of these types of fibers in real field scenarios.

\section{THEORY}

In Raman DTS systems based on the principle of optical time domain reflectometry (OTDR), a short and intense laser pulse is launched into the sensing fiber. The light pulse propagates along the fiber, interacting with the molecules that compose the optical fiber core [13]. This interaction generates light scattering, which can be both elastic and inelastic. The scattering is elastic when the frequency of the scattered light is equal to the frequency of the light source, i.e Rayleigh [14]. The other type of scattering occurs when the frequency of the light source differs from the frequency of the scattered signal. This is the case of Brillouin and Raman scatterings [15].

A small portion of the scattered light is captured and guided backwards along the fiber towards the end where the pulse is launched. Through a circulator, the backscattered signals are guided into filters to separate the Raman backscattered light from the Rayleigh and Brillouin backscattered signals. Finally, the filtered signals are detected by photodiodes, which are typically high-sensitivity low-noise avalanche photodiodes (APD) because the power of the collected signals is very small (60-70dB below the used peak power levels [16]). The output signals are sampled using a high-resolution analog-to-digital conversion (ADC) system.

Once the sampling time and the speed of light in the fiber are known, it is possible to calculate the position $\mathrm{z}$ in the fiber where the scattering has occurred. The spatial resolution depends on the pulse width of the injected laser pump. To achieve $1 \mathrm{~m}$ spatial resolution (resolution commonly used in practical applications), pulses with 10ns duration are required [17]. The pulse repetition frequency is limited by the fiber length [18]. The maximum frequency is given by the following equation: $2 \mathrm{~nL} / \mathrm{c}$, where $\mathrm{n}$ is the refractive index of the core, $\mathrm{L}$ is the fiber length and $\mathrm{c}$ is the speed of light in vacuum.

In the Raman scattering process, the frequency shifts depend on the characteristic vibrational frequencies of the molecules. The backscattered Raman upshifted frequency component is termed anti-Stokes line (AS) and the downshifted component is called Stokes line (S). The Raman frequency shift in fused silica is approximately $\Delta \mathrm{f}=13.2 \mathrm{THz}(\sim 100 \mathrm{~nm}$ for wavelength $1550 \mathrm{~nm}$ ) [19]. The intensity of the AS backscattered exhibits a strong dependence on temperature $(\sim 0.8 \% / \mathrm{K})$, while the $\mathrm{S}$ backscattered is only slightly temperature dependent $(\sim 0.1 \% / \mathrm{K})[20]$.

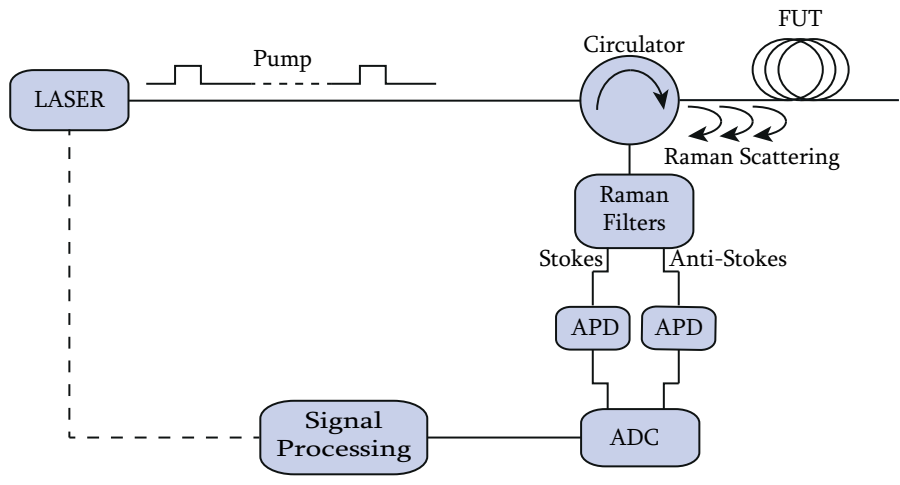

Fig. 1. Schematic diagram of Raman distributed temperature sensor system based on OTDR technique.

Figure 1 shows a typical schematic of Raman DTS system based on OTDR. This system exploits the strong dependence on the AS component with temperature to estimate it. However, the AS backscattered signal depends not only on the temperature, but also on the fiber attenuation at AS wavelength and local losses (such as splices, connectors and bending losses). Variations in local losses can be misinterpreted as variations in temperature. To solve this problem, the AS signal is normalized with another backscattered signal (Rayleigh or Stokes component).

For a single-ended Raman DTS measurement using both components of the Raman scattering, the temperature profile along the fiber can be given from the power ratio of the antiStokes $\left(\mathrm{P}_{A S}\right)$ and stokes $\left(\mathrm{P}_{S}\right)$ lines, according to equation [21]:

$$
T(z, t)=\frac{\gamma}{\ln \left[\frac{P_{S}(z, t)}{P_{A S}(z, t)}\right]+C-\int_{0}^{z} \Delta \alpha\left(z^{\prime}\right) d z^{\prime}},
$$

where $\mathrm{T}$ is the absolute temperature, $\mathrm{z}$ is the distance along the fiber and $\mathrm{C}$ is a dimensionless constant parameter which groups the influence of various factors such as the difference in the photodetector responsibility at the two wavelengths. In this work, the constant $\mathrm{C}$ has been considered invariant over time given that the DTS system temperature does not change. Van de Giesen et al. reported that $\mathrm{C}(\mathrm{t})$ is dependent on the instrument temperature and it is not a function of position along the cable [21]. $\gamma$ is defined as $h \Delta v / K$ with $\mathrm{K}$ being the Boltzmann's constant, h the Planck's constant and $\Delta v$ the separation between Raman anti-Stokes/Stokes and probe light frequencies.

Finally, the term $\int_{0}^{z} \Delta \alpha\left(z^{\prime}\right) d z^{\prime}$ stands for the accumulative differential attenuation of the Stokes and anti-Stokes backscatter light along the fiber. In standard fibers, this parameter can be determined through a calibration. For this purpose, it is necessary to maintain a section of the fiber in a stable and known temperature (assuming that the value of this term does not present significant changes along the fiber). The double-ended calibration can also be used when $\Delta \alpha$ presents a non-uniform special profile, but it has to be assumed that this parameter does not change over time [21]. Normally this calibration process is carried out once and before the measurements. 


\section{EXPERIMENTAL SET-UP}

In order to carry out a comparison between the use of standard multimode fibers and gold-coated multimode fibers to perform distributed temperature measurements, the experimental set-up shown in Figure 2 has been implemented.

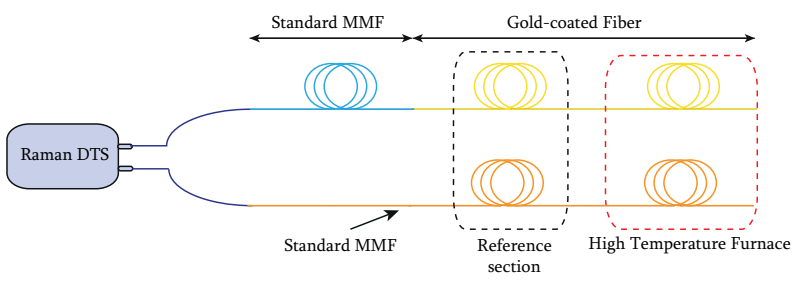

Fig. 2. Schematic diagram of the RTDS system used in the experimental tests: Raman-based Distributed Temperature Sensor instrument (RDTS). sensing fiber employed, on the one hand, $8 \mathrm{~m}$ of standard multimode graded index fiber (blue) and gold-coated fiber of 79m length (yellow), on the other hand, $33 \mathrm{~m}$ of standard graded-index multimode fiber (orange).

A Distributed Temperature Sensor (DTS) interrogator based on Raman Optical-Time-Domain-Reflectometry (ROTDR) was used to accomplish the measurements. The Raman DTS source is provided by a laser operating at $1064 \mathrm{~nm}$ with an average output power of $17 \mathrm{~mW}$. The laser beam waist diameter and the numerical aperture are approximately $50 \mu \mathrm{m}$ and $0.2 \mathrm{re}-$ spectively.

The fiber under test (FUT) used in the set-up consisted in, on the one hand, $8 \mathrm{~m}$ of standard graded-index multimode fiber (MMF-50/125 $\mu \mathrm{m}$ ) spliced to 79m gold-coated fiber-pure fused silica core (FiberGuide AFS50/125/155G). The use of standard MMF is to facilitate access to the gold-coated fiber. On the other hand, $33 \mathrm{~m}$ of standard graded-index multimode fiber (MMF-50/125 $\mu \mathrm{m})$. The last $5 \mathrm{~m}$ of both type of FUTs were inserted into a high temperature controlled chamber. The two FUTs were connected to two channels of the RDTS system.

The furnace temperature was modified between room temperature $\left(20^{\circ} \mathrm{C}\right.$ approximately) and $600^{\circ} \mathrm{C}$ with steps of $50^{\circ} \mathrm{C}$. After changing the temperature and before taking the measurements, enough time was considered until the temperature was stable in the furnace chamber.

For the calibration process, $20 \mathrm{~m}$ of gold-coated fiber and same length of standard multimode fiber (reference section) has been used. This section has been maintained at an uniform and stable temperature. The calibration parameters obtained were used to accomplish all measures. Each temperature measurement has been repeated 6 times under the same conditions. The time used to carry out each temperature measurement was 60 s.

\section{RESUlts}

The anti-Stokes and Stokes signals traces obtained from the RDTS instrument for the standard multimode fiber using a $\mathrm{R}=0.5 \mathrm{~m}$ spatial resolution are shown in Figure 3 . The standard MMF has been introduced with its coating in the furnace chamber. It can be clearly noticed the increase of the temperature values leading to an increase of the power in both Anti-Stokes and Stokes signals recovered from the hot-spot

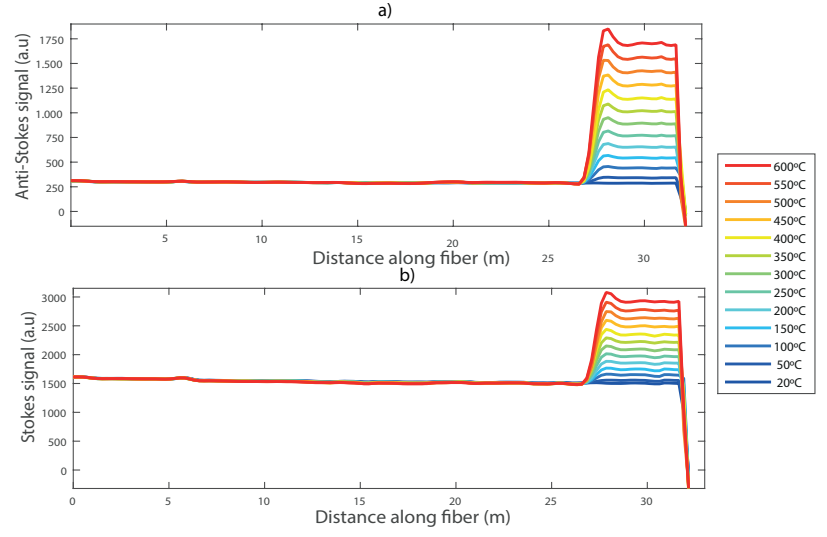

Fig. 3. a) Anti-Stokes and b) Stokes signals obtained for the standard multimode fiber.

section. The anti-Stokes component presents a larger increase with temperature in comparison with the Stokes component.

In addition, it can also be observed that in the start of the section where the temperature changes, the traces present peaks in the form of "S". This fact could be because of the chromatic dispersion effect [21].

On the other hand, when the temperature was increased (to more than $300^{\circ} \mathrm{C}$ ), the cover disappeared. However, this fact has not introduced significant changes in terms of losses in the AS and S signals. In this sense, and in order to see a comparison in the behavior between uncoated and coated fibers in a Brillouin-based distributed sensor system, $\mathrm{Xu}$ et al. (2016) compared both fibers in a range of temperatures from $25^{\circ} \mathrm{C}$ to $1100^{\circ} \mathrm{C}$. This work concluded that the difference throughout the temperature range was approximately $4^{\circ} \mathrm{C}$. This could probably be attributed only to the difference of strain suffered by both fibers [22]. This fact does not affect the studies presented in this work given that the Raman effect is insensitive to strain.

Figure 4 shows the same signals previously discussed, but now the traces have been obtained from the FUT containing gold-coated fiber. It can be seen that in the first $8 \mathrm{~m}$, which corresponds to the standard MMF, both the anti-Stokes and Stokes signals have recovered present more power than the recuperated by the pure silica core gold-coated fiber at room
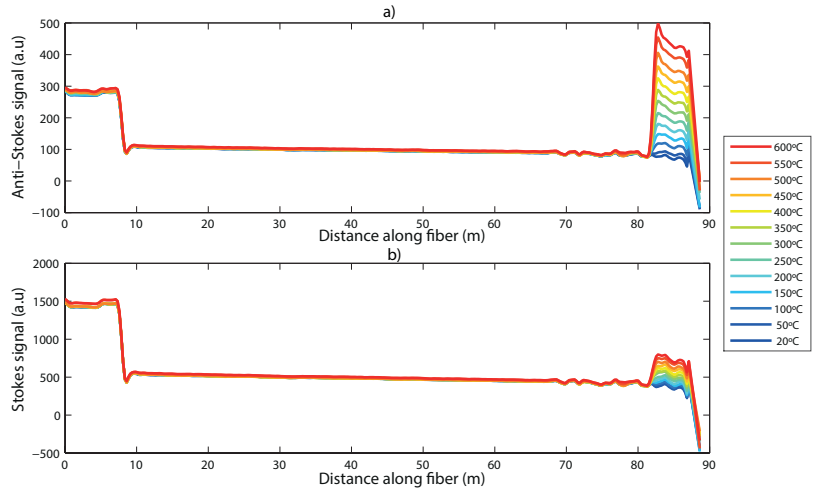

Fig. 4. a) Anti-Stokes and b) Stokes signals obtained for the gold-coated fiber. 
temperature $\left(20^{\circ} \mathrm{C}\right.$ approximately). The power decay of the backscattered signals is due to, on the one hand, the local losses produced in the fusion of the standard MMF fiber and the gold coated fiber and, on the other hand, to the backscattered signals generated in pure fused silica fibers that are less than the signals generated in the standard MMF. The Raman cross section in the pure silica fibers is significantly smaller than in the Ge doped fibers [19].

It can also be observed a non-uniform profile and small variations in the traces of the Raman backscattered signals in the hot zone. This is owing to struts losses introduced by the microbending induced by the metal coating [11].

From these recovered Raman backscattered signals in both FUTs, the temperature profile along the two channels has been estimated. In order to see the influence of the losses variation with temperature in the gold-coated fiber, the calibration parameters obtained at room temperature have been used for calculating the temperature in all measurements. Figure 5 shows the temperature estimated in the hot-spot section for the two FUTs.

The error committed in the first 8 meters (corresponding to pigtail) is large, $5^{\circ} \mathrm{C}$ approximately. This error has been calculated as the difference between room temperature and the average of all the measurements in this section. This is because the estimated temperature for these $8 \mathrm{~m}$ has been determined with the configuration parameters obtained after the calibration with the gold fiber. These parameters are different to those obtained when the calibration is performed with the pigtail section.

As can be observed, using the ratio of the anti-Stokes to Stokes signals for determining the temperature, it can be corrected the local loss variations discussed previously in the Raman backscattered signal traces. However, in the hot-spot section the non-uniform special profile of the losses in the gold-coated fiber induces errors in the temperature estimated. Table 1 shows the average temperature of the section submitted
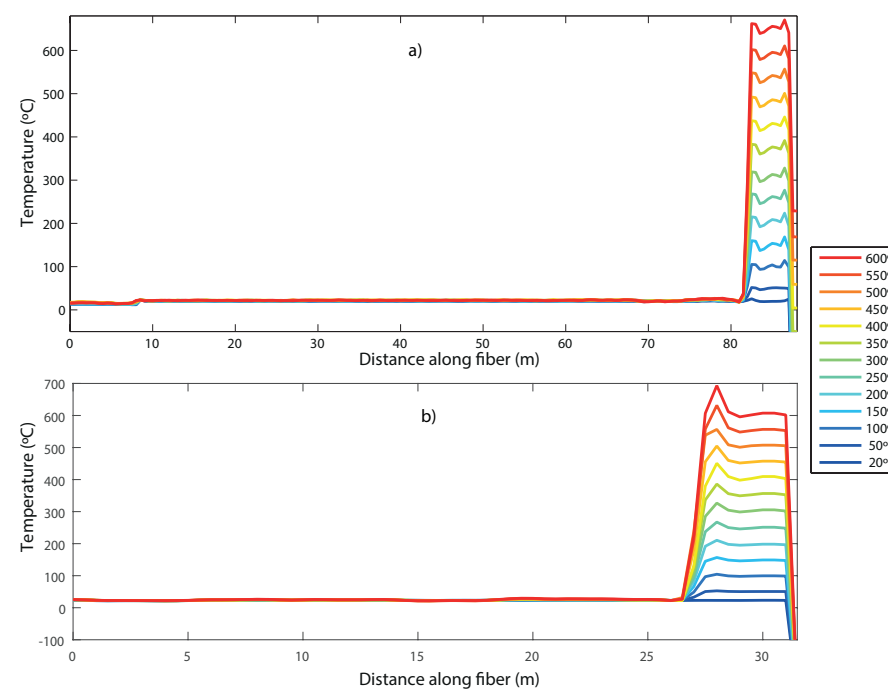

Fig. 5. Temperature profile (a) FUT containing the gold-coated fiber (b) FUT with standard MMF only. to temperature variations and its associated error for the two types of fiber. The error has been calculated as the absolute value of the difference between the real furnace temperature and the average temperature of the hot-spot section.

TABLE I

THE AVERAGED TEMPERATURE VALUES AND THE MEAN ERRORS FOR THE GOLD-COATED FIBER (COLUMN 2 AND 3) AND THE STANDARD MMF (COLUMN 4 AND 5).

\begin{tabular}{ccccc}
\hline \hline Temperature $\left({ }^{\circ} \mathrm{C}\right)$ & T. $\left({ }^{\circ} \mathrm{C}\right)$ & Error $\left({ }^{\circ} \mathrm{C}\right)$ & T. $\left({ }^{\circ} \mathrm{C}\right)$ & Error $\left({ }^{\circ} \mathrm{C}\right)$ \\
\hline 20 & 19.54 & 0.46 & 22.97 & 2.97 \\
50 & 49.52 & 0.48 & 50.55 & 0.55 \\
100 & 98.59 & 1.41 & 98.99 & 1.01 \\
150 & 146.80 & 3.2 & 147.98 & 2.02 \\
200 & 201.81 & 1.81 & 197.08 & 2.92 \\
250 & 254.99 & 4.99 & 249.12 & 0.88 \\
300 & 306.07 & 6.07 & 302.99 & 2.99 \\
350 & 369.84 & 19.84 & 353.88 & 3.88 \\
400 & 424.19 & 24.19 & 405.44 & 5.44 \\
450 & 479.00 & 29.00 & 455.94 & 5.94 \\
500 & 535.03 & 35.03 & 505.88 & 5.88 \\
550 & 588.61 & 38.61 & 554.84 & 4.82 \\
600 & 648.70 & 48.70 & 604.25 & 4.25 \\
\hline \hline
\end{tabular}

From the results presented in Table 1, it can be noticed that, under the same conditions, the committed errors using the gold-coated fiber are much higher than those obtained with standard multimode fiber. The measurement error in the pure silica gold-coated fiber increases as temperature rises. The procedure of calibration and measurement used, can be valid in the case of the multimode fibers. However, in the case of the gold-coated fiber this process cannot be applied. A dynamic calibration is necessary to compensate the variations in the profile, because this type of fibers have a temperature dependent loss. A previous study of loss variations as a function of temperature in these fibers [11] has demonstrated that in $10 \mathrm{~m}$ of gold-coated fiber it is possible to obtain up to $20 \mathrm{~dB} / \mathrm{Km}$ difference in the losses between the low and high temperatures. In addition, the attenuation does not maintain the same spectrum for all wavelengths. Therefore, the value of the accumulative differential attenuation is changing as the temperature is being modified.

Added to these problems, the modification of the channel configuration and the hysteresis losses exhibited by the goldcoated fibers, can change the value of the accumulative differential attenuation. Hence, it is not possible to calibrated the Raman DTS system for each temperature and save the calibration parameters for future measurements. Taking into account these considerations, it is necessary to realize a calibration process after each change in temperature. The procedure to carry out the dynamic calibration needed in these cases, is explained later.

To achieve measurements in real environment field, it can contemplate the use of a section of the fiber subjected to a uniform and similar temperature required to perform the calibration process. In this case, the calibration parameters obtained (including the differential attenuation) will be similar to those needed to accomplish a correct measurement. Nevertheless, this solution cannot be implementable at all times. Providing high and stable temperatures in an additional reference section is really complex in some environments. 
Another alternative is the use of the same section where the temperature is monitored to carry out the calibration process. This solution is applicable to environments where the temperatures to be measured are stable and uniform. This option reduces the added complexity of installing other systems for calibration. Here, it can detect dynamically sections subjected to different temperatures and calculate the calibration parameters for each section separately.

To carry out the calibration process proposed in this work, it was necessary to determine that the temperature is stable along entire channel. To achieve this goal temperature measurements were performed periodically and the measured temperature was compared with the previously calculated. If the difference between both measurements was low (less than a predetermined threshold) it would be considered that the temperature profile was stable and therefore the calibration process could be performed. This procedure is valid for systems where the temperature changes slowly.

Once this test was realized, the channel was divided in various sections, corresponding to different types of fibers or sections under different temperatures. This division could be performed manually, when the properties of the channel to measure were known, or automatically detecting changes in temperature, or in anti-Stokes/Stokes traces. Here, an algorithm used in image processing for edge detection was employed. In this work, the first derivative of the temperature trace was used and those areas where it was not equal to zero were searched. After delimiting those areas, it proceeded to calculate the calibration parameters $(\gamma, \mathrm{C}$ and $\Delta \alpha)$ needed to obtain a correct temperature profile using Equation 1.

Firstly, the differential attenuation, $\Delta \alpha$, was determined for the reference section. The value of this parameter was calculated independently of $\mathrm{C}$ and $\gamma$. Moreover, it is not necessary to know the exact temperature value of the section where it was estimated. Only through the powers of both antiStokes and Stokes signals recovered from two separate points at the same temperature $\left(z_{1} \mathrm{y} z_{2}\right), \Delta \alpha$ is determined by [23]:

$$
\Delta \alpha\left(z_{1}, z_{2}\right)=\frac{\ln \left[\frac{P_{S}\left(z_{1}\right)}{P_{A S}\left(z_{1}\right)}\right]-\ln \left[\frac{P_{S}\left(z_{2}\right)}{P_{A S}\left(z_{2}\right)}\right]}{z_{2}-z_{1}}
$$

However, in this work, in order to improve the accuracy of this parameter determination, a section at uniform temperature was considered instead of individual points. In this fiber section, the AS and S signals decay exponentially under the form [24]:

$$
P_{S / A S}(z)=P_{0 S / 0 A S} \mathrm{e}^{\left(-\alpha_{S / A S} z\right)}
$$

where $P_{0 S}$ and $P_{0 A S}$ are the power of the AS and $\mathrm{S}$ signals, at the scatter point respectively. Taking the natural logarithm of the ratio between the two signals, the following expression is obtained:

$$
\ln \left[\frac{P_{S}(z)}{P_{A S}(z)}\right]=\ln \left[\frac{P_{0 S}(z)}{P_{0 A S}(z)}\right]+\Delta \alpha z,
$$

This equation shows that $\ln \left[P_{S} / P_{A S}\right]$ varies linearly with distance z. Hence, $\Delta \alpha$ was calculated as the slope of a fitted line of the recovered points of $\ln \left[P_{S} / P_{A S}\right]$ from this section.
Once this parameter was calculated, it was possible to determine the other two using the stable and known temperature of the reference section (Figure 2). Fixing $\gamma$ to its theoretical value, $\gamma=\mathrm{h} \Delta \mathrm{v} / \mathrm{k}=490 \mathrm{~K}$ [21] for a laser emitting in $1064 \mathrm{~nm}$, the constant $\mathrm{C}$ was determined divesting it of Equation 1.

As previously mentioned, the parameters $\gamma$ and $\mathrm{C}$ were considered constants and invariant over time. Therefore, it has only been necessary to determinate $\Delta \alpha$ for the other sections. Finally, the value of $\int_{0}^{z} \Delta \alpha\left(z^{\prime}\right) d z^{\prime}$ in the position $\mathrm{z}$ was calculated as the sum of each $\Delta \alpha$ of the different sections until the position $\mathrm{z}$.

In this work, the channel has been divided into 3 main sections. The first one, corresponding to the $8 \mathrm{~m}$ of the standard $\mathrm{MMF}$, the second section is corresponding to the gold-coated fiber submitted at room temperature and finally, the hot spot section (third section). For the first two sections, calibration parameters have not changed because the temperature and the channel configuration were the same. Therefore, the temperatures obtained in these two sections for all the measurements were the same as those shown above. For the hot-spot section, the system is calibrated after each change in temperature. Figure 6 presents the temperature of this section calculated after applying the corrections in the values of the accumulative differential attenuation in each temperature.

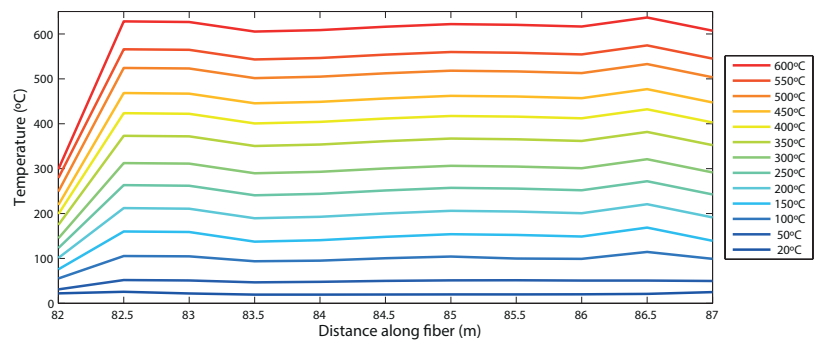

Fig. 6. Temperature profile after correction the accumulative differential attenuation.

It can be seen that the temperature measured by this method gives better results in comparison with the conventional calibration process. The measurement error has been reduced to $5^{\circ} \mathrm{C}$ approximately at $600^{\circ} \mathrm{C}$.

\section{Conclusions}

This work arises from the need to solve the measurement problems in temperatures higher than $300^{\circ} \mathrm{C}$ because the standard fibers can not be used. In these cases, the acrylate coating disappears, and therefore the fiber could become more fragile and prone to suffer irreversible damage. Therefore, in this temperature range, is necessary to employ metal-coated fibers to give them the required protection in order to maintain its physical and optical properties. A high-temperature distributed sensing system based on a ROTDR implementation and two types of fibers: a standard multimode fiber and multimode gold-coated fiber have been presented in this paper. In addition, a comparison between these fibers have been discussed. A calibration technique has been implemented to obtain experimental temperature measurements independently of the dynamic variation of the optical loss with the temperature in 
the Stokes and the anti-Stokes wavelengths. This allows real implementation in environments where a high and dynamic temperatures is required. The measured temperature range was between room temperature $\left(20^{\circ} \mathrm{C}\right.$ approximately) and $600^{\circ} \mathrm{C}$ with steps of $50^{\circ} \mathrm{C}$.

\section{ACKNOWLEDGMENT}

This work has been supported by ENSA through TOMATIN project (cofounded by the Cantabria government) and by the Spanish Ministry of Economy and Competitiveness through the projects TEC2013-47264-C2-1-R and TEC2016-76021C2-2-R.

\section{REFERENCES}

[1] S. Adachi, "Distributed optical fiber sensors and their applications," SICE Annual Conference, IEEE, pp. 329-333, 2008.

[2] I. Toccafondo, T. Nannipieri, A. Signorini, E. Guillermain, J. Kuhnhenn, M. Brugger, and F. Di Pasquale, "Raman distributed temperature sensing at cern," Photonics Technology Letters, IEEE, vol. 27, no. 20, pp. 21822185,2015

[3] J.M. Lopez-Higuera, L. Rodriguez Cobo, A.Q. Incera, and L.R. Cobo, "Fiber optic sensors in structural health monitoring," Light wave Journal of Technology, vol. 29, no. 4, pp. 587-608, 2011.

[4] E. Udd, "Fiber optic sensors," SPIE Optical Engineering Press, 1993.

[5] X. Bao, and L. Chen, "Recent progress in distributed fiber optic sensors," Sensors vol. 12, no. 7, pp. 8601-8639, 2012.

[6] A. Ukil, H. Braendle, and P. Krippner, "Distributed temperature sensing review of technology and applications," Sensors Journal, IEEE, vol. 12, no. 5, pp. 885-892, 2012.

[7] G. Bolognini, and A. Hartog, "Raman-based fibre sensors: Trends and applications," Optical Fiber Technology, vol. 19 no. 6, pp. 678-688, 2013.

[8] G.R. Williams, G. Brown, W. Hawthorne, A.H. Hartog, and P.C. Waite, "Distributed temperature sensing (DTS) to characterize the performance of producing oil wells," Environmental and Industrial Sensing International Society for Optics and Photonics vol. 39, pp. 39-54, 2000.

[9] G.O. Fridleifsson, and W.A. Elders, "The Iceland Deep Drilling Project: a search for deep unconventional geothermal resources." Geothermics, vol. 34, no. 3, pp. 269-285, 2005.

[10] A. Méndez, and T.F. Morse, "Specialty optical fibers handbook." Academic Press, 2011.

[11] T. Reinsch, and J. Henninges, "Temperature-dependent characterization of optical fibres for distributed temperature sensing in hot geothermal wells," Measurement Science and Technology, vol. 21, no. 9, pp. 094022, 2010.

[12] S. Mandal, S. Dekate, B. K. Lee, R. Guida, M. Mondanos, J. Yeo and M. Goranson, "Characterization and calibration of Raman based distributed temperature sensing system for $600^{\circ} \mathrm{C}$ operation,"SPIE Sensing Technology+ Applications. International Society for Optics and Photonics,pp. 94910A-94910A8, 2015

[13] J.P. Dakin, D.J. Pratt, G.W. Bibby, and J.N. Ross, "Distributed optical fibre Raman temperature sensor using a semiconductor light source and detector," Electronics letters, vol.21 no. 13, pp. 569-570, 1985.

[14] L. Palmieri, and L. Schenato "Distributed Optical Fiber Sensing Based on Rayleigh Scattering," The Open Optics Journal, vol.7, pp. 104-127, 2013.

[15] P.C. Wait, K. De Souza, K. and T.P. Newson, "A theoretical comparison of spontaneous Raman and Brillouin based fibre optic distributed temperature sensors," Optics communications, vol. 144 no.1-3, pp. 17-23, 1997.

[16] M.A. Soto, T. Nannipieri, A. Signorini, A. Lazzeri, F. Baronti, R. Roncella, and F. Di Pasquale, "Raman-based distributed temperature sensor with $1 \mathrm{~m}$ spatial resolution over $26 \mathrm{~km}$ SMF using low-repetitionrate cyclic pulse coding," Optics letters, vol. 36 no.13, pp. 2557-2559, 2011.

[17] L. Yuan, L. Tao, W. Yubin, S. Zhihui, W. Chang, and L. Tongyu, "Application of distributed optical fiber temperature sensing system based on Raman scattering in coal mine safety monitoring," Photonics and Optoelectronics (SOPO) Symposium on IEEE, pp. 1-4, 2012.

[18] F. Baronti, A. Lazzeri, R. Roncella, R. Saletti, A. Signorini, M. A Soto and F. Di Pasquale, "SNR enhancement of Raman-based long-range distributed temperature sensors using cyclic Simplex codes," Electronics letters, vol. 46, no. 17 pp. 1221, 2010.
[19] M. A. Farahani, and T. Gogolla, 1999. "Spontaneous Raman scattering in optical fibers with modulated probe light for distributed temperature Raman remote sensing," Journal of Lightwave Technology, vol. 17, no.8, pp. 1379-1391, 1999.

[20] A. H. Hartog, A.P. Leach and M.P. Gold, "Distributed temperature sensing in solid-core fibres," Electronics letters, vol. 21, no 23, pp. 1061$1062,1985$.

[21] N. Van De Giesen, S.C. Steele-Dunne, J. Jansen, O. Hoes, M.B. Hausner, S. Tyler and J. Selker, "Double-ended calibration of fiber-optic Raman spectra distributed temperature sensing data,"Sensors, vol. 12 no. 5, pp. 5471-5485, 2012.

[22] P. Xu, Y. Dong, D. Zhou, C. Fu, J. Zhang, H. Zhang, Z. Lu, L. Chen and X. Bao, " $1200 \mathrm{C}$ high-temperature distributed optical fiber sensing using Brillouin optical time domain analysis," Applied Optics, vol. 55 no. 21, pp. 5471-5478, 2016.

[23] F. Surez, J. E. Aravena, M. B. Hausner, A. E. Childress and S. W. Tyler, "Assessment of a vertical high-resolution distributed-temperature-sensing system in a shallow thermohaline environment," Hydrology and Earth System Sciences, vol. 15 no. 3, pp. 1081-1093, 2011.

[24] M. B. Hausner, F. Surez, K. E. Glander, N. V. D. Giesen, J. S. Selker and S. W. Tyler, "Calibrating single-ended fiber-optic Raman spectra distributed temperature sensing data," Sensors, vol. 11 no. 11, pp. 1085910879, 2011.

Ismail Laarossi was born in 1987. He obtained the Telecommunication Technical Engineering Degree (Electronic Systems) in 2011, the degree in Telecommunication Engineering in 2013 and the Physics, Instrumentation and environment Master Degree in 2014, at the University of Cantabria (Spain). $\mathrm{He}$ is currently doing his $\mathrm{PhD}$ thesis and collaborating in the Photonics Engineering Group, in the area of distributed temperature sensing, especially those based on Raman effect.

Ruben Ruiz Lombera received the degree in Telecommunication Technical Engineering (Electronic Systems) in 2011, the degree in Telecommunication Engineering in 2012, and the Master degree in Information Technologies and Mobile Networks from the University of Cantabria (Spain). He is currently doing his $\mathrm{PhD}$ in the area of distributed fiber sensors, especially those based on Brillouin scattering.

Maria Angeles Quintela was born in Santander, Spain, in 1974. She received both the Telecommunications Technical Engineering degree and the Telecommunications Engineering degree from the University of Cantabria, Spain. In 1998, she joined the University of Cantabria as a Lecturer. Her teaching topics include electronic components and optical communications. In 2005, she received the Ph.D. degree from the University of Cantabria. Her main research is on computer modelling and experimental characterisation of fiber lasers. She has co-authored more than 60 papers, presented in conferences and scientific journals.

Jesus Mirapeix received the masters degree in telecommunications engineering in 2000, and the Ph.D. degree with a focus on quality monitoring of welding processes by means of plasma optical spectroscopy, in 2007. Since 2012, he has been an Associate Professor with the Photonics Engineering Group, Department of Electronic Technology, Industrial Automation and System Engineering, University of Cantabria, with teaching and R\&D duties. He is currently involved in distributed sensor systems based on stimulated Brillouin scattering. He is the Co-Founder of SADIQ Engineering, a company offering products and services for welding, automation, and monitoring. His research interests include fiber optic sensors and processing strategies for online quality assurance of both arc and laser welding processes, with application in the manufacturing of components for the aerospace and nuclearsectors. 
Domingo Lima was born in October 1972 in Sal-Cabo Verde. He obtained the Industrial Technical Engineering degree from the University of Cantabria (Spain). In 2003 he joined the Equipos Nucleares (ENSA) company. Currently, he is the Head of Advanced Technology Center of ENSA.

David Solana was born in November 1972. He obtained the Mining TECHNICAL ENGINEERING, specializing in metallurgy, from the University of Cantabria (Spain) in 1997. In 1998 he joined the Equipos Nucleares (ENSA) company. Since 2003, he has been Welding Technician responsible.

Jose Miguel Lopez Higuera (M93SM98) was born in February 1954, in the village of Ramales de la Victoria, Cantabria, Spain. He obtained the Telecommunication Technical Engineering degree from the Universidad Laboral de Alcala de Henares, Madrid, Spain and the Telecommunication Engineering degree from the Universidad Politecnica de Madrid (UPM), Madrid, Spain. He received the Ph.D. degree in telecommunication engineering, with an extraordinary award, from UPM. He founded and is the Head of the Photonics Engineering Group of the TEISA Department in the University of Cantabria. Currently, he works in the development of Photonics Instrumentation, photonic/optical fibre sensor systems for civil engineering, electrical power, environmental and smart structures and for optical diagnostics for a wide range of applications. He has directed more than $50 \mathrm{R} \& \mathrm{D}$ projects and has written or co-written more than 400 publications in the form of books, chapters of books, papers and conferences, both national and international, and obtained ten patents. He is the Editor and Co-author of several books, including i) Optical Sensors, UC, 1998; ii) the Handbook of Photonic Sensing Technology,Wiley and Sons, New York, NY, USA, 2002 and iii) he is the co-editor of the book Engineering a High-Tech Business: Entrepreneurial experiences and Insights, published by SPIE-Press, USA, 2008. Prof. Lopez-Higuera is Senior Member of the IEEE and member of the IEE, SPIE and OSA. 